\title{
LAS CONSECUENCIAS DE LA GLOBALIZACIÓN EN EL DERECHO CONSTITUCIONAL: APORTACIONES DE LA MIRÍADA DE CORRIENTES DOCTRINALES
}

The consequences of globalization for constitutional law: Contributions of the myriad doctrinal currents

\author{
NAIARA ARRIOLA ECHANIZ \\ Universidad de Deusto, Bilbao \\ naiara.arriola@deusto.es
}

Cómo citar/Citation

Arriola Echaniz, N. (2017).

Las consecuencias de la globalización en el derecho constitucional: aportaciones de la miríada de corrientes doctrinales. Revista de Estudios Políticos, 178, 171-188. doi: https://doi.org/10.18042/cepc/rep.178.06

\section{Resumen}

El fenómeno de la globalización ha sido capaz de desbordar los contenedores nacionales surgidos en el mundo después de la Segunda Guerra Mundial para convertirnos en ciudadanos de una aldea con problemas de carácter global ante los que un único Estado o una determinada organización internacional se tornan instrumentos de solución obsoletos e insuficientes. En este sentido, el presente trabajo pretende analizar el concepto de globalización, desde una perspectiva jurídico-política, y cómo esta afecta al derecho constitucional en relación con la democracia, la tutela de los derechos humanos y el poder económico de los Estados.

\section{Palabras clave}

Globalización; derecho constitucional; Estado; Unión Europea; organizaciones internacionales.

\section{Abstract}

The phenomenon of globalization goes beyond the national borders established after the Second World War, generating a global village with problems that cannot be 
solved by a single State or a specific international organization. This paper analyses globalization from a politico-legal perspective, to understand its effects on constitutional law in relation to democracy, the guaranteeing of Human Rights, and national economic powers.

\section{Keywords} izations.

Globalization; constitutional law; State; European Union; international organ- 


\section{SUMARIO}

I. INTRODUCCIÓN: LA GLOBALIZACIÓN COMO FENÓMENO JURÍDICO-POLÍTICO EN PROCESO DE CONSTITUCIONALIZACIÓN. II. LA DEMOCRACIA ANTE LA GLOBALIZACIÓN. III. LA PROTECCIÓN MULTINIVEL DE LOS DERECHOS HUMANOS. IV. LA INTERACCIÓN ENTRE LA GLOBALIZACIÓN Y EL PODER ECONÓMICO DE LOS ESTADOS. V. EPÍLOGO. BIBLIOGRAFía.

\section{INTRODUCCIÓN: LA GLOBALIZACIÓN COMO FENÓMENO JURÍDICO-POLÍTICO EN PROCESO DE CONSTITUCIONALIZACIÓN}

La globalización pudo tener su origen con la mundialización de la economía y del comercio, pero en la actualidad es un fenómeno que ha sido capaz de desbordar los contenedores nacionales e internacionales surgidos en el mundo después de la Segunda Guerra Mundial para convertirnos en ciudadanos de una aldea con problemas de carácter global ante los que un único Estado o una determinada organización internacional se tornan instrumentos de solución obsoletos e insuficientes (Holton y Turner, 2016). Se trata de un proceso de intensificación de las dependencias recíprocas que no se reducen al mercado mundial (Beck, 1999). La globalización se define como un fenómeno multinivel en el que los Estados ya no dominan a las sociedades nacionales delimitadas por el principio de la territorialidad, sino que hoy se impone la diferenciación funcional de una única sociedad a escala mundial (Teubner, 1997: 22).

La corriente cosmopolita ha profundizado en el estudio de la globalización defendiendo la repolitización de las nuevas estructuras de poder que, en la actualidad, superan al Estado nacional en algunos de sus ámbitos competenciales. El ordenamiento jurídico debe rebasar el nivel nacional, en la regulación de aquellos ámbitos en los que éste sea disfuncional, en su control o en su gestión, y apoyarse en nuevas fuentes de legitimidad, bien en organizaciones locales, regionales, internacionales o supranacionales. Así, esta teoría debe entenderse como una forma o una posible vía para enfrentarse y superar tanto la anomia de la globalización económica imperante, cuanto los efectos negativos que esta conlleva, no solo para las instituciones políticas, nacionales y/o internacionales, sino también para los individuos, el medio ambiente, la sanidad y la cultura, entre otros (Beck, 2002). Este planteamiento 
defendido por Beck (2005) nos aleja del realismo político y del nacionalismo metodológico.

El nuevo orden mundial globalizado, la fragmentación del derecho, el reparto asimétrico de poder entre los Estados que conforman la comunidad internacional y la incapacidad de las organizaciones internacionales para asegurar los valores y derechos recogidos en las constituciones a ese nivel demandan una revisión del proceso mismo de constitucionalización, que racionalizó los Estados desde el surgimiento de las primeras constituciones, para que se encuentren los canales para aproximar y adaptar ese proceso al nuevo panorama supranacional que, de facto, ya ha absorbido muchas de las competencias y de los poderes estatales.

En términos estatistas, Gordillo y Martinico (2015) señalan que la constitucionalización supone la existencia de dos procesos. Por un lado, la constitucionalización de un Estado se puede dar desde una perspectiva externa. Esta forma de constitucionalización supone la ordenación de las normas internas y la depuración de los sistemas de solución de conflictos. El desarrollo del Estado de derecho y la existencia de un sistema de fuentes del derecho que ordenen todas las normas jurídicas dentro de un Estado constituyen las bases de esta constitucionalización externa. Por otro lado, la constitucionalización de un Estado se puede producir por la humanización del mismo (constitucionalización interna). Este proceso supone el reconocimiento de unos derechos fundamentales para los ciudadanos y la garantía de los mismos frente al poder del Estado. Dentro de este proceso de humanización de los Estados, las sociedades han ido alcanzando históricamente cada vez más cotas de derechos. En el Estado liberal se garantizó una libertad mínima frente al Estado, unos derechos basados en la economía de mercado y en la propiedad privada, pero con la aparición del Estado del bienestar dichos derechos se ampliaron y el Estado promovió desde entonces el respeto y la garantía de derechos de tipo asistencial para con sus ciudadanos.

El derecho constitucional se está desarrollando más allá del horizonte nacional. Bajo esta premisa, Gordillo y Martinico (2013: 429-478) analizan cómo se desarrollan procesos de constitucionalización más allá del Estado. Por un lado, la constitucionalización externa de las organizaciones internacionales se produce para regular la fragmentación del derecho internacional, en un sentido de federalización. Esta forma de constitucionalización supone un intento de gobernar los ordenamientos jurídicos a escala global y, como consecuencia de lo anterior, de estabilizar las relaciones entre la multitud de ordenamientos jurídicos existentes. Por otro lado, la constitucionalización interna de las organizaciones internacionales se basa en la humanización de las mismas. En este proceso se busca reforzar la garantía y el respeto de los derechos de los individuos a escala internacional. Los derechos humanos pasan a 
formar parte del derecho internacional y las organizaciones internacionales tratan de garantizar determinados derechos individuales según su ámbito sectorial de actuación. La progresiva humanización supone la afirmación de los derechos humanos a escala supranacional para disciplinar el mercado internacional.

El nuevo panorama globalizado incide en la distribución del poder político y lleva a una relación más estrecha la cooperación internacional del primer tercio del siglo xx. El factor interestatal del poder internacional está siendo sustituido por una mayor cooperación y dependencia mutua a escala global. A continuación, nos centraremos en los tres aspectos en que se pueden observar las consecuencias de la globalización en el derecho constitucional: la participación democrática, la tutela supranacional de los derechos humanos y los nuevos centros de regulación del poder económico estatal.

\section{LA DEMOCRACIA ANTE LA GLOBALIZACIÓN}

En términos generales, la democracia apunta a la legitimación del poder político, a la participación de los ciudadanos en la toma de decisiones y, en última instancia, al control y la exigencia de responsabilidad política a los gobernantes. En la actualidad, la democracia conlleva la admisión del sufragio universal, libre, igual y directo de quienes componen una comunidad política, de manera que el principio democrático cumple una función legitimadora del poder político, que solo es reputado legítimo si cuenta con el consentimiento de los ciudadanos. Pero, además, el principio democrático implica que los ciudadanos participan indirectamente (salvo cuando lo hacen directamente a través del referéndum o de la iniciativa legislativa popular) en la toma de decisiones políticas a través de representantes, dando origen a la democracia representativa. Estos representan al conjunto de la comunidad política, no a cada uno de sus miembros, lo cual nos sitúa ante el importante concepto de la representación política y el punto clave para el funcionamiento de todo sistema democrático: la elección de representantes (Friedrich, 1972: 13-38).

García Guerrero (2017) analiza el embate que la globalización origina a la democracia. Para ello, analiza la afección a la democracia de las dos fases de la globalización. La primera fase de la globalización abarca desde la caída del Muro de Berlín hasta la creación del Mercado Único Europeo. La segunda fase de la globalización se caracteriza por el cambio de sus actores, los Estados, a los ejecutivos de las integraciones económicas supraestatales. Las consecuencias constitucionales van in crescendo cuando la unión aduanera se encamina al mercado común, y alcanzan su plenitud en la segunda fase de la globalización. En esta última fase, la integración es más profunda que en la primera y 
la democracia se ve afectada por la creación de un órgano más o menos ejecutivo, por la implementación legislativa que exigen y por la creación de órganos arbitrales o judiciales. En estas integraciones —en el mejor ejemplo posible: la Unión Europea-, la ciudadanía solo participa muy indirectamente a través de su Parlamento nacional y del europeo. No obstante, por la propia lógica de funcionamiento y de influencia de cada Estado en la arena internacional, el ataque a la democracia no afecta por igual a todos.

No obstante, García Guerrero (2017: 138-143) circunscribe su investigación a las consecuencias que las dos etapas de la globalización tienen sobre la democracia representativa en los Gobiernos parlamentarios. En este escenario, hay razones para pensar que tanto la globalización como la Unión Europea se inspiran en el ordoliberalismo: economía de mercado basada en la libre competencia, estabilidad de las divisas, libre comercio, garantías a la propiedad privada, libertad contractual, responsabilidad de los actores económicos y, por último, continuidad de la política económica. Por un lado, en la primera etapa de la globalización, ese poder económico respondería al ordoliberalismo. Ante esta situación, el derecho constitucional debería exigirle al poder económico la igualdad en las condiciones básicas de ejercicio de la actividad económica para reconstituir el principio democrático. Habría que reforzar el control del Parlamento sobre el Ejecutivo, así como el control de los Parlamentos nacionales sobre sus respectivos Ejecutivos. Cuando se mantengan negociaciones con otros Gobiernos de la integración, se requerirán partidos políticos que se correspondan con el ámbito de integración, así como comisiones parlamentarias específicas, dotarse de un auténtico Parlamento bicameral y, finalmente, la aprobación de una constitución federal sometida a referéndum del pueblo que corresponda con el nuevo Estado. Por otro, en la segunda etapa de la globalización, se aumenta la intensidad de la integración. Por tanto, esta parece que recomienda que el tratado internacional constitutivo se adopte con cuórums equivalentes a los de una reforma constitucional, y que sean sometidos a referéndum. Además, se deben generar controles de los Parlamentos nacionales o de los Parlamentos bicamerales para controlar las negociaciones del Ejecutivo o del Gobierno de la integración, respectivamente.

\section{LA PROTECCIÓN MULTINIVEL DE LOS DERECHOS HUMANOS}

Los derechos humanos suponen el reconocimiento de una esfera de autonomía individual frente a la injerencia de los poderes públicos. Hoy está asentada la idea de que toda constitución debe contener una declaración formal de derechos. A pesar de ello, la doctrina difiere considerablemente a la hora de determinar cuáles merecen la consideración de «fundamentales». De todas 
formas, se acostumbra a distinguir entre los derechos que tratan de configurar parcelas inaccesibles al poder, de inspiración liberal, y los derechos que apuntan a la legitimación, participación y control del poder por los ciudadanos, de inspiración democrática (García Pelayo, 1991: 353). López Guerra (2013: 136-139) clasifica los derechos contenidos en la Constitución Española en atención a la garantía, a la naturaleza y al contenido. En el presente apartado, siguiendo esta última clasificación, se presentarán los mecanismos de tutela supranacionales de los mismos: europeo e iberoamericano. Y, por último, se aportará una reflexión sobre la incipiente defensa de derechos por parte de determinadas organizaciones internacionales.

En primer lugar, Gordillo (2015) dirige una obra colectiva que reflexiona sobre los recientes desarrollos y retos de la constitucionalización de los tribunales europeos supranacionales, entendiendo por tales el Tribunal de Justicia de la Unión Europea y el Tribunal Europeo de Derechos Humanos. El compendio de trabajos de investigación que se recogen en este texto pone de manifiesto cómo la tutela de derechos dentro de la Unión Europea ha avanzado más allá de los tribunales constitucionales nacionales, por ejemplo: en relación con el concepto de la dignidad humana (Oehling, 2015: 21-32), el derecho a la educación (Álvarez, 2015: 33-30). No obstante, también se destacan como retos a los que se enfrenta esta tutela supranacional de derechos los siguientes: el triángulo problemático que se genera entre las constituciones nacionales, el Convenio Europeo de Derechos Humanos y el derecho de la Unión tras la entrada en vigor del Tratado de Lisboa (Jimena y Tajadura, 2015; Martinico, 2015: 193-216) y la adhesión de la propia Unión al convenio (Matia, 2015: 217-241); la ejecución de las sentencias del Tribunal Europeo de Derechos Humanos en el ordenamiento jurídico español (Milone, 2015: 81-105), y la necesidad de aumentar los recursos del citado tribunal (Hernández: 2015: 107-136).

Asuntos como Kadi ponen de manifiesto cómo los retos destacados en el sistema de tutela desarrollado dentro de la Unión necesitan también de una convergencia no solo europea sino también dentro del sistema de las Naciones Unidas (Gordillo, 2017b; Kumm, 2011). No obstante, como señala López Castillo (2017), las controversias sobre la interpenetración pacífica de normas constitucionales entre la Unión Europea y sus Estados miembros se manifiestan en algunos asuntos, sirviendo como botón de muestra el análisis de la Sentencia de la Sala Segunda del Tribunal Constitucional Federal Alemán (TCFA) de 15 de diciembre de 2015.

En segundo lugar, observando la actuación de la Corte Interamericana de Derechos Humanos, se está desarrollando una protección supranacional de derechos en Iberoamérica. Sin embargo, antes de reseñar las bondades de la corte, es importante tener presente que este segundo sistema de tutela 
supranacional de derechos cuenta con dificultades en la ejecución de sus sentencias ya que los Estados americanos ratificaron el Pacto de San José con efectos jurídicos diferentes y, por tanto, la uniformidad que existe en el sistema europeo no es trasladable al sistema que se abordará a continuación (Arias, 2012).

Carmona (2015) dirige una obra en la que se compara la protección de los derechos humanos desde una perspectiva de género en ambos sistemas judiciales. Esta perspectiva metodológica sitúa el foco de análisis de los derechos humanos en las desigualdades entre hombres y mujeres y en la discriminación sufrida por estas últimas. No obstante, no solo se analiza la casuística derivada de ambos sistemas, sino que se aportan líneas de acción y de regulación para la posible superación de la desigualdad de género. La discriminación por razón de género impregna multitud de aspectos de nuestros ordenamientos jurídicos. Sin embargo, un análisis desde una perspectiva nacional de esta situación reduce las posibilidades de tutela de los derechos de las mujeres, porque la protección de los derechos humanos no solo reside en los tribunales nacionales, sino que también debería regularse en los tratados internacionales de protección de los derechos humanos, a saber: el Convenio Europeo de los Derechos Humanos y el Pacto de San José. El Tribunal Europeo de Derechos Humanos y la Corte Interamericana de Derechos Humanos se erigen como instituciones fundamentales para alcanzar una tutela efectiva de los derechos humanos desde una perspectiva de género. La incorporación de la perspectiva de género a los sistemas europeo e interamericano de derechos humanos se ha producido recientemente y, en general, sin una referencia expresa a la misma. La discriminación de género presenta una carencia estructural. Al no existir derechos específicos en este ámbito, se produce la dependencia de las mujeres del activismo judicial para la garantía de los derechos, con la consecuente falta de seguridad jurídica que se da en ambos sistemas normativos. La revisión de los pronunciamientos del Tribunal Europeo de Derechos Humanos y de la Corte Interamericana de Derechos Humanos en esta materia pone de manifiesto la falta no solo de una normativa internacional, sino de legislaciones nacionales con una perspectiva de género. En el sistema europeo, la protección del Tribunal Europeo de Derechos Humanos parece que solo conecta violencia de género con discriminación en algunos países. Sin embargo, la Corte Interamericana de Derechos Humanos ha sido, en general, más avanzada en la aplicación de la perspectiva de género a la violencia contra las mujeres, pero, sobre todo, hay que destacar el papel de la Comisión Interamericana de Derechos Humanos. El sistema interamericano ha desarrollado un conjunto de estándares con el objetivo de prevenir, investigar y sancionar la violencia contra las mujeres. Esta violencia se puede dar en multitud de supuestos que son analizados, lo que permite conocer la amplitud del problema que nos ocupa y, sobre todo, permite extraer pautas de acción aplicables por el 
Tribunal Europeo de Derechos Humanos para una mejor protección de la violencia contra las mujeres.

Por último, Petersmann (2017) reflexiona sobre la defensa de derechos por parte de determinadas organizaciones internacionales y sobre la necesidad de un cambio en la propia teoría clásica del derecho internacional. En el ámbito concreto de la Organización Mundial del Comercio, el citado autor (2008: 955-960) defiende que la OMC garantiza la libertad, la no discriminación y el Estado de derecho más allá de las constituciones nacionales en muchos países donde tendía a estar limitada la libertad económica de sus ciudadanos y donde los productos, los bienes y los servicios extranjeros estaban discriminados. Extendiendo la igualdad de libertades a través de las fronteras y controlando las políticas comerciales extranjeras a restricciones jurídicas y judiciales, las reglas de la Organización Mundial del Comercio sirven a «funciones constitucionales" para establecer los derechos humanos y las correspondientes obligaciones gubernamentales en el ámbito de la política comercial. Para Petersmann (2002), los derechos humanos tienen un papel fundamental, y entre ellos se ubica también la libertad de comercio. Dentro de esta corriente de pensamiento, la inclusión de derechos internacionales no comerciales en los procesos de solución de diferencias ejemplifica la manera en que los Estados, las escuelas y los órganos del Sistema de Solución de Diferencias han fortalecido la sustancia constitucional de la Organización. La afirmación de que la OMC no puede evitar cuestiones medioambientales y de derechos humanos se ha convertido en una verdad y ha provocado una modificación en la forma en la que se entiende su proceso de constitucionalización. Esto ha derivado en la inclusión de temas medioambientales y otros temas no comerciales en la Ronda de Doha, así como el reconocimiento de ciertos valores no comerciales por su Órgano de Solución de Diferencias.

Tanto si la solución a esta cuestión es expandir la constitución sustantiva de la Organización Mundial del Comercio como reducirla, la verdad es que las intersecciones entre comercio y derechos humanos son inevitables y producen entendimientos particulares en los ámbitos de acción de la Organización y en las elecciones que están disponibles para ella (Lawrence, 2013: 88). En esta línea, Joerges y Petersmann (2011) destacan, sobre todo, el hecho de que una constitución consiste en un preacuerdo en torno a las normas que efectivamente van a constituir los derechos civiles y limitar los poderes del Estado. En este contexto, se consideran fundamentales los derechos denominados como market freedoms o economic freedoms. Sin embargo, Dunoff y Trachtman (2009: 188-189) señalan que el derecho de la Organización Mundial del Comercio no incluye un conjunto de derechos fundamentales, y mucho menos un derecho fundamental a la libertad de comercio. En este punto se menciona la diferencia EE. UU.-artículos 301 a 310 de la Ley de Comercio Exterior de 1974 (DS152), 
ya que en su solución se produjo el debate más relevante en torno a si la Organización establecía un derecho individual a la libertad de comercio, así como si producía efecto directo como ordenamiento jurídico. Los citados autores concluyen señalando que ni los textos de la Organización Mundial del Comercio ni las recomendaciones y resoluciones de su Sistema de Solución de Diferencias recogen referencias a la garantía de derecho individuales.

La revisión desarrollada en el presente apartado, siguiendo los conceptos dados por Gordillo y Martinico (2013), muestra cómo la globalización está sufriendo un proceso de constitucionalización desde su perspectiva interna, desarrollándose instituciones de tutela de los derechos humanos más allá del Estado. No obstante, esta humanización constante del derecho internacional pone de manifiesto, como ha señalado reiteradamente Gordillo (2017a, 2012a y 2012b), la falta de un ordenamiento unitario y de un sistema integrado de tutela de los derechos humanos.

\section{LA INTERACCIÓN ENTRE LA GLOBALIZACIÓN Y EL PODER ECONÓMICO DE LOS ESTADOS}

Los problemas actuales producen efectos más allá de las fronteras nacionales y necesitan de una acción política conjunta para su gestión, por ejemplo, en el ámbito de la seguridad y la economía, la regulación laboral y el medio ambiente, entre otros (Holton y Turner, 2016). El término «agenda global» se utiliza para aglutinar de forma genérica todos aquellos problemas que han surgido derivados de la globalización y que no se pueden resolver de forma autónoma por los distintos Estados nacionales, sino que necesitan de la cooperación transnacional. Las consecuencias negativas de la globalización se pueden entender incluidas en la agenda global cuando su problemática se haya visto como un objeto que necesita de la acción política para su gestión y/o solución. Held (2005: 132) acuña el concepto de problemas «intermésticos» para referirse al incremento de problemas que para su gestión y solución necesitan la combinación de política nacional y política internacional.

En el ámbito estrictamente económico, la globalización de los flujos comerciales también ha puesto en evidencia el surgimiento de problemas globales. Como máximo exponente de ello destacamos la crisis financiera global que se inició en 2008 y que ha llevado a los Estados a poner en común su poder económico y promover un debate en torno a la necesidad de controlar y regular la globalización económica para evitar, en la medida de lo posible, sus consecuencias negativas.

En el contexto globalizado, el papel del Estado para regular los flujos económicos, aunque es central, ya no tiene la exclusividad que tuvo en épocas 
anteriores. Sassen (Krasner et al., 2010: 128-134) propone el concepto de «autoridad privada» para referirse al componente del nuevo orden institucional y privatizado mediante el cual se gobierna y organiza la economía global. La autoridad privada constituye una forma extremadamente parcial de regulación en el mundo actual.

Las consecuencias más destacables de la acción de estas autoridades privadas son: 1) en primer lugar, el crecimiento de actividades transfronterizas y de la acción de los sujetos globales que operan fuera del sistema interestatal formal afecta a la competencia y al ámbito de actuación de los Estados y del derecho internacional clásico; 2) en segundo lugar, el hecho de que este dominio se esté institucionalizando y sometiendo cada vez más al desarrollo de mecanismos de gobernanza privados afecta a la exclusividad de la autoridad del Estado y del derecho internacional (exclusividad que siempre tuvo un alcance limitado); 3) en tercer lugar, los crecientes poderes normativos en esta esfera privada afectan al poder normativo del derecho internacional; 4) en cuarto lugar, la participación del Estado en esta nueva regulación de su propio papel en la economía y la incipiente desnacionalización de algunos de sus elementos institucionales, necesarios para permitir el funcionamiento de algunas de las nuevas políticas asociadas a la globalización, transforman aspectos fundamentales del Estado. Al hacerlo alteran la estructura organizativa que permite exigir responsabilidad pública en los Estados y también la estructura organizativa del sistema interestatal y del propio derecho internacional.

Con el objetivo de demostrar los flujos que se generan como consecuencia de la globalización y el poder económico de los Estados se van a tomar como ejemplos: por un lado, la gobernanza económica existente dentro de la Unión Europea, y, por otro, la interconexión normativa que se está produciendo entre la propia Unión Europea, sus Estados miembros y la Organización Mundial del Comercio.

Por un lado, el proceso de integración de la Unión Europea ha tenido como consecuencia la creación de un poder económico con capacidad de gobierno, entendida esta en términos constitucionales, independiente de sus Estados miembros. El primer hito importante lo encarna la creación del Mercado Común Europeo, ya que, aunque algunas otras organizaciones internacionales han intentado alcanzar este estadio, la Unión ha sido la única capaz de alcanzarlo (González, 2016). De hecho, la Unión Europea ha profundizado su integración económica constituyendo lo que García Guerrero y Martínez Alarcón califican como «unidad económica supra estatal» (2016: 16). Esta forma de concebir la Unión Europea ha permitido analizar de manera multidisciplinar las afecciones que este proceso ha acometido respecto del concepto racional normativo de constitución. Concluyendo que estas no se limitan a ciertas partes de las constituciones de los Estados miembros, sino a la práctica totalidad de las 
normas fundamentales. Este hecho tiene como consecuencias cuatro problemas centrales: 1) la desconstitucionalización económica de los Estados miembros de una manera asimétrica y progresiva; 2) la adopción constitucional de la estabilidad presupuestaria; 3) la necesidad de emplear como elemento de mejora de la competitividad la materia tributaria; y 4) las políticas de austeridad de la Unión que restringen los valores constitucionales, los derechos sociales, los derechos de los trabajadores y la protección del medio ambiente, entre otros (García Guerrero y Martínez Alarcón, 2016: 19-20).

De los cuatro problemas destacados, Martínez Alarcón y Lagos Rodríguez (2017) analizan el aspecto concreto de la estabilidad presupuestaria debido a que, durante la crisis, la Unión Europea ha actuado básicamente para garantizar la solidez de la moneda, y ello ha requerido profundizar en el proceso de integración económica. Sin embargo, los nuevos desarrollos de la gobernanza económica europea han fortalecido el sesgo intergubernamental en el funcionamiento de la Unión, en detrimento de la publicidad y la transparencia, dificultando, a su vez, la rendición de cuentas. Todo ello en detrimento de la existencia de un poder europeo legítimo y limitado. Desde el punto de vista económico, la regla de la estabilidad presupuestaria, tal y como se ha configurado en su aplicación en un periodo de crisis económica, ha generado una pérdida de las convergencias de las economías y de la cohesión del territorio, con el consiguiente riesgo de desintegración. El escenario que se plantea es «constitucionalmente desalentador» debido a que los últimos desarrollos señalados han alterado su arquitectura institucional, minimizando el rol de los canales comunitarios y fortaleciendo de forma evidente su sesgo intergubernamental, y esto se ha hecho, en general, sin compensar esta tendencia con un reforzamiento del papel de las instituciones representativas en el nivel nacional como instrumentos de control efectivo de lo que sus Ejecutivos hacen en las instituciones europeas. En este contexto, Martínez Alarcón y Lagos Rodríguez (2017: 182) entienden que un reforzamiento intenso del papel de los Parlamentos nacionales no compensa la falta de legitimidad democrática en el nivel europeo, donde decisiones fundamentales para los ciudadanos se estarían adoptando de espaldas a los requerimientos básicos del principio democrático. La constitucionalización de la regla de la estabilidad presupuestaria supone, además, un recorte de la capacidad de poder de los Estados, debido a que actualmente su única opción para reducir la presión fiscal e incrementar la renta disponible de los agentes económicos privados es una severa restricción del gasto social que permita equilibrar el resultado final del presupuesto (Martínez Alarcón y Lagos Rodríguez, 2017: 184).

Por otro lado, en el ámbito de las organizaciones internacionales, se plantea como reto para el derecho constitucional la asunción por parte de estas de esferas propias del poder económico estatal. En el apartado anterior se ha 
puesto de manifiesto cómo la Organización Mundial del Comercio ha tutelado determinados derechos y valores constitucionales en su sistema de solución de controversias y también los ha incorporado en su propio derecho. En este momento, revisando cómo se ha globalizado el poder económico de los Estados, centramos, de nuevo, el foco de análisis en la citada organización. Se puede comenzar defendiendo que la actividad de la Organización Mundial del Comercio está generando la convergencia entre su derecho y el ordenamiento jurídico de la Unión Europea. En un análisis de la interconexión del sistema de fuentes del derecho de los ordenamientos jurídicos de dicha organización y la Unión, en particular en lo que respecta a las diferencias comerciales sobre las que encontramos pronunciamientos tanto de los Grupos Especiales y del Órgano de Apelación de la Organización Mundial del Comercio como del propio Tribunal de Justicia de la Unión Europea, se puede afirmar que el nivel de fricción entre el derecho de la OMC y el derecho de la UE, generado como consecuencia del cumplimiento y la aplicación de las recomendaciones y resoluciones adoptadas por el Órgano de Solución de Diferencias, se reducirá a medida que se clarifique el complejo sistema de fuentes del derecho (Arriola, 2017).

Por medio de un análisis empírico del cumplimiento de las recomendaciones y resoluciones adoptadas en el marco del Sistema de Solución de Controversias Comerciales de la Organización, se pueden extraer las líneas generales de relación o interconexión entre ambos sistemas institucionales y jurídicos que permitan extrapolar los sistemas de fuentes de derecho nacionales a este escenario. No obstante, la convergencia es incipiente y no exenta de problemas. Así, Bronkers (2008) señala que en la práctica parece haber esfuerzos por poner el derecho de la Unión de conformidad con ellos, y de esta forma les da cumplimiento. Indudablemente, al no emitir una declaración explícita de su posición ante dichas obligaciones internacionales, las instituciones europeas se guardan el derecho de separarse de las decisiones adoptadas en el seno de la OMC en caso de considerarlo conveniente. Cass (2005) contrargumenta, ante la actitud de la Unión Europea en algunas controversias comerciales, que la actividad de la Organización Mundial del Comercio es una fuerza dinámica constitucionalizadora en virtud de su capacidad de generar normas y estructurar las ya existentes durante la solución de una diferencia. Según el caso controvertido, esta constitucionalización se genera mediante cuatro distintos procesos: 1) toma en préstamo de reglas constitucionales, principios y doctrinas de ordenamientos jurídicos externos al derecho de la propia organización, para resolver las diferencias comerciales; 2) elaboración de normas respecto de la carga de la prueba; 3 ) entrada de la organización a solucionar problemas entendidos tradicionalmente dentro del ámbito la soberanía estatal, como la salud pública; y 4) por último, inclusión entre sus 
argumentos de valores constitucionales ajenos a lo estrictamente comercial. En definitiva, se está construyendo un sistema constitucional mediante interpretaciones judiciales que emanan de la institución judicial de solución de diferencias de la Organización Mundial del Comercio.

Desde una perspectiva más general, Trachtman (2014) continúa con este debate y defiende la necesidad de constitucionalizar el derecho de la Organización Mundial del Comercio y, por extensión, el derecho internacional, y concluye sus análisis afirmando que el sistema legal internacional tiene una constitución e incluye dentro de esta a la constitución de la Organización Mundial del Comercio, y que aquella tiene rasgos vis-à-vis con los Estados, con otras organizaciones internacionales y con el sistema legal internacional en general.

Como se ha puesto de manifiesto, en el contexto globalizado, el papel del Estado para regular los flujos económicos, aunque es central, ya no tiene la exclusividad que tuvo en épocas anteriores. De hecho, en el contexto de la Unión Europea, es inconcebible que los Estados puedan actuar de manera independiente y, de hecho, el proceso de integración demuestra que los Estados no solo no pueden hacerlo, sino que no quieren. Solo resta confiar en que los restos pendientes se aborden con mayor inclusión de los valores y elementos propios del derecho constitucional, para aumentar la legitimidad de sus instituciones, y que se refuercen los derechos sociales.

En el ámbito del sistema multilateral de comercio, la Organización Mundial del Comercio y su sistema de solución de controversias comerciales, en la línea presentada en este apartado, deberán seguir impulsando la cohesión normativa en este ámbito. Esta cohesión promueve una interconexión constitucionalizada de los ordenamientos jurídicos de los miembros de la citada organización, siguiendo una rudimentaria jerarquía normativa. Esta perspectiva puede servir como línea abierta de investigación para la constitucionalización de la globalización económica en otras organizaciones internacionales, así como para promover un sistema de gobernanza global, al menos, en este campo.

\section{EPÍLOGO}

El nuevo panorama globalizado incide en la distribución del poder político y lleva a una relación más estrecha a la cooperación internacional del primer tercio del siglo xx. El factor interestatal del poder internacional está siendo sustituido por una mayor cooperación y dependencia mutua a escala global. Este artículo ha propuesto una revisión de los avances que se están produciendo en la constitucionalización de la globalización, en el ámbito de la democracia, la tutela de los derechos humanos y la regulación del poder económico; pero también se han tratado de destacar multitud de interrogantes y 
líneas de investigación pendientes de abordar por la doctrina constitucionalista, tanto en estos tres ámbitos concretos como en otros que se han plasmado inconclusos en estas páginas.

En la actualidad, la democracia conlleva la admisión del sufragio universal, libre, igual y directo de quienes componen una comunidad política, de manera que el principio democrático cumple una función legitimadora del poder político, que solo es reputado legítimo si cuenta con el consentimiento de los ciudadanos. Sin embargo, esta democracia no solo se enfrenta a críticas dentro de la esfera nacional, sino que la ciudadanía también reclama que los centros decisorios supranacionales de poder implementen formas de participación democrática, con el objetivo principal de que sus decisiones cuenten con mayor legitimidad y de que sus actuaciones puedan sujetarse a algún tipo de responsabilidad.

En el campo de la garantía de los derechos humanos, el Tribunal Europeo de Derechos Humanos, el Tribunal de Justicia de la Unión Europea y la Corte Interamericana de Derechos Humanos se erigen como instituciones fundamentales para alcanzar una tutela efectiva de los mismos. No obstante, la falta de una normativa constitucional supranacional, bien europea, bien iberoamericana, similar a los sistemas de fuentes nacionales, genera controversias y retos para la garantía efectiva de los derechos, sobre todo en la efectiva ejecución de las sentencias dictadas por dichos tribunales supraestatales.

Por último, los nuevos centros de regulación del poder económico supranacionales muestran cómo el derecho constitucional debería adaptarse a este nuevo ámbito. Como se ha puesto de manifiesto, en el contexto globalizado, el papel del Estado para regular los flujos económicos, aunque es central, ya no tiene la exclusividad que tuvo en épocas anteriores. La Unión Europea, en este campo concreto, ha mostrado cómo superar los contenedores nacionales y poder manejar la economía nacional desde la esfera supranacional. No obstante, esto no debe tener como consecuencia que los valores constitucionales que socializan la economía permanezcan al margen de este proceso y queden relegados a las constituciones nacionales donde ya no tiene efectividad.

En la esfera estrictamente internacional, la Organización Mundial del Comercio impulsa la cohesión normativa dentro del sistema multilateral del comercio. Esta cohesión genera una interconexión de los ordenamientos jurídicos de los miembros de la citada organización. Sin embargo, dicha interdependencia normativa reclama una regulación que organice la aplicación de los distintos ordenamientos jurídicos. La propuesta es generar un sistema de fuentes del derecho similar a las nacionales para evitar las antinomias jurídicas que surgen como consecuencia de la actividad de esta organización. Esta demanda puede servir, además, como impulso de la investigación que genere una constitucionalización de la globalización económica global. 


\section{Bibliografía}

Álvarez Álvarez, H. (2015). Democratic Education and the Normative Power of the Factual. En L. I. Gordillo Pérez (dir.). Constitutionalism of European Supranational Courts. Recent developments and challenges (pp. 33-30). Pamplona: Thomson Reuters Aranzadi.

Arias López, B. W. (2012). Fundamentos de la obligatoriedad de las Sentencias de la Corte Interamericana de Derechos Humanos. Lex social: Revista de los Derechos Sociales, 1, 74-94.

Arriola Echaniz, N. (2017). La convergencia de dos sistemas de integración: las relaciones entre la OMC y la UE en perspectiva constitucional. Pamplona: Thomson Reuters Aranzadi.

Beck, U. (1999). What is Globalization? Cambridge: Polity Press.

- (2002). Libertad o capitalismo. Conversaciones con Johannes Willms. Barcelona: Paidós. (2005). La mirada cosmopolita o la guerra es la paz. Barcelona: Paidós.

Bronkers, M. (2008). From «Direct Effect» to «Muted Dialogue». Journal of Ineternational Economic Law, 4 (11), 885-898.

Carmona Cuenca, E. (ed.) (2015). La perspectiva de género en los Sistema Europeos e Interamericano de Derechos Humanos. Madrid: Centro de Estudios Políticos y Constitucionales.

Cass, D. Z. (2005). The Constitucionalization of World Trade Organization. Legitimacy, Democracy, and Community in the International Trading System. Oxford, New York: Oxford University Press.

Dunoff, J. L. y Trachtman, J. P. (eds.) (2009). Ruling the World? Constitutionalism, International Law, and Global Governance. Cambridge: Cambridge University Press.

Friedrich, C. J. (1972). Gobierno constitucional y democracia. Teoría y práctica en Europa y América. Vol. II. Madrid: Instituto de Estudios Políticos.

García Guerrero, J. L. (2017). Los embates de la globalización a la democracia. Revista de Estudios Politicos, 176, 113-146.

- y Martínez Alarcón, M. L. (2016). Constitución y mercado en la crisis de la Unión Europea: consideraciones preliminares. Estudios de Deusto, 64 (1), 15-21.

García Pelayo, M. (1991). Obras completas. Madrid: Centro de Estudios Constitucionales.

González Jiménez, M. (2016). El Mercado Común. Estudios de Deusto, 64 (1), 137-164.

Gordillo Pérez, L. I. (2012a). Interlocking Constitutions. Towards an Interordinal Theory of National, European and UN Law. UK: Hart Publishing.

- (2012b). Constitución y ordenamientos supranacionales. Madrid: Centro de Estudios Políticos y Constitucionales.

- (dir.) (2015). Constitutionalism of European Supranational Courts. Recent Developments and Challenges. Pamplona: Thomson Reuters Aranzadi.

- (2017a). Understanding the Current Fragmentation of the Law and the Coexistence of Supranational Legal Orders. Anuario Iberoamericano de Justicia Constitucional, 21 [en prensa].

- (2017b). El TJUE y el Derecho internacional: la defensa de su propia autonomía como principio. Cuadernos de Derecho Transnacional, 9 (2) [en prensa].

- y Martinico, G. (2013). La jurisprudencia federalizante y humanizadora del Tribunal de Justicia. Un cuento desde el país de las hadas. Teoría y Realidad Constitucional, 32, 429-478. 
Held, D. (2005). Un pacto global. La alternativa socialdemócrata al consenso de Washington. Madrid: Santillana Ediciones Generales.

Hernández Ramos, M. (2015). The Collapse of the Constitutional Courts in Protecting Fundamental Rights. The European Court of Human Rights in Comparative Perspective. En L. I. Gordillo Pérez (dir.). Constitutionalism of European Supranational Courts. Recent Developments and Challenges (pp. 107-136). Pamplona: Thomson Reuters Aranzadi.

Holton, R. J. y Turner, B. S. (eds.) (2016). The Routledge International Handbook of Globalization Issues. London, New York: Routledge.

Jimena Quesada, L. y Tajadura Quesada, J. (2015). La prehistoria y la historia del derecho constitucional europeo (ensayo de paralelismo con el decurso del Derecho Constitucional nacional). Revista de Derecho Político, 94, 11-52.

Joerges, C. y Petersmann, E. U. (2011). Multilevel Trade Governance in the WTO Requires Multilevel Constitutionalism. Oxford, Portland, Oregon: Hart Publishing.

Krasner, S. D., Sassen, S. y Teubner, G. (2010). Estado, soberanía y globalización. Bogotá: Siglo del Hombre Editores.

Kumm, M. (2011). How Does European Union Law Fit into the World of Public Law? Costa, Kadi and Three Models of Public Law. En N. Jurgen y A. Wiener (eds.). Political Theory of the European Union (pp. 111-147). Oxford, New York: Oxford University Press.

Lawrence, J. C. (2013). Contesting constitutionalism: Constitutional discourse at the WTO. Global Constitutionalism, 2 (1), 63-90.

López Castillo, A. (2017). ¡Alto ahí a la «identidad constitucional»!: Un ejemplo (ya no tan) reciente de discontinuidad continuista en la jurisprudencia iuscomunitaria del TCFA. Teoria y Realidad Constitucional, 39, 387-412.

López Guerra, L. (2013). Derecho Constitucional. El ordenamiento constitucional. Derechos y deberes de los ciudadanos. Valencia: Tirant lo Blanch.

Martínez Alarcón, M. L. y Lagos Rodríguez, M. G. (2017). La gobernanza económica de la Unión durante la crisis: consecuencias de un proceso de la integración. Revista de Estudios Politicos, 176, 147-189.

Martinico, G. (2015). The Problematic Triangle: National Constitutions, the ECHR and EU Law in the Post Lisbon Scenario. En L. I. Gordillo Pérez (dir.). Constitutionalism of European Supranational Courts. Recent developments and challenges (pp. 193-216). Pamplona: Thomson Reuters Aranzadi.

Matia Portilla, F. J. (2015). La adhesión de la UE al CEDH hoy: una valoración de los trabajos realizados hasta el momento. En L. I. Gordillo Pérez (dir.). Constitutionalism of European Supranational Courts. Recent Developments and Challenges (pp. 217-241). Pamplona: Thomson Reuters Aranzadi.

Milone, C. (2015). «Algo queda por hacer...» La ejecución de las Sentencias del Tribunal Europeo de Derechos Humanos en el ordenamiento jurídico español. En L. I. Gordillo Pérez (dir.). Constitutionalism of European Supranational Courts. Recent developments and challenges (pp. 81-105). Pamplona: Thomson Reuters Aranzadi.

Oehling de los Reyes, A. (2015). The Human Dignity Concept in the Case Law of the European Court of Human Rights. En L. I. Gordillo Pérez (dir.). Constitutionalism of European Supranational Courts. Recent Developments and Challenges (pp. 21-32). Pamplona: Thomson Reuters Aranzadi. 
Petersmann, E. U. (2002). Time for a United Nations «Global Compact» for Integrating Human Rights into the Law of Worldwide Organizations: Lessons from European Integration. European Journal of International Law, 13 (3), 621-650.

- (2008). Human Rights, International Economic Law and Constitutional Justice: A Rejoinder. European Journal of International Law, 19 (5), 955-960.

- (2017). Multilevel Constitutionalism for Multilevel Governance of Public Goods. Methodology Problems in International Law. UK: Hart Publishing.

Teubner, G. (1997). Global Bukowina: Legal Pluralism in the World Society. En G. Teubner (ed.). Global Law without a State (pp. 3-31). Dartmounth: Aldershot.

Trachtman, J. P. (2014). The Future of International Law. Global Government. Cambridge: Cambridge University Press. 